\title{
Comparison of olfactometrically detected compounds and aroma properties of four different edible parts of Chinese mitten crab
}

\author{
Siru $\mathrm{Ji}^{1} \cdot$ Saiqi $\mathrm{Gu}^{2} \cdot$ Xichang Wang ${ }^{1} \cdot \mathrm{Na} \mathrm{Wu}^{1}$
}

Received: 1 May 2015 / Accepted: 29 August 2015 / Published online: 24 September 2015

(c) The Author(s) 2015. This article is published with open access at Springerlink.com

\begin{abstract}
Chinese mitten crab Eriocheir sinensis from the Songjiang district in Shanghai is popular among Chinese consumers due to its unique pleasant aroma. In this study, volatile compounds in four edible parts of steamed male E. sinensis were extracted by the monolithic material sorptive extraction method and analyzed by gas chromatography-mass spectrometry/olfactometry, the E-Nose technique, and sensory evaluation. Results showed that 2 , 7, 7 and 10 important odor compounds (IOCs, odor intensity value $\geq 3$ ) were determined in abdomen, claw, leg meat, and gonad parts, respectively. Among them, 2-ethylpyridine (fishy odor) was the only IOC in all four parts. Benzaldehyde (almond odor) and trimethylamine (fishy odor) were common to claw meat, leg meat, and gonad parts but not found as the IOCs in abdomen meat. Besides, 3-methyl-2-thiophenecarboxaldehyde (chocolate odor) and 2-acetylthiazole (roast meat odor) were the IOCs found exclusively in abdomen meat and gonad parts, respectively. There existed major differences between gonad parts and 3 types of crabmeat. Sensory evaluation results showed that meaty aroma was the prominent aroma of abdomen meat
\end{abstract}

Siru Ji and Saiqi Gu contributed equally to this work and are considered co-first authors.

Saiqi Gu

Xichang Wang

xcwang@shou.edu.cn

1 College of Food Science and Technology, Shanghai Ocean University, No. 999, Hucheng Huan Road, Lingang New City, Shanghai 201306, China

2 Ocean College, Zhejiang University of Technology, No. 18, Chaowang Road, Hangzhou 310014, China and claw meat. Leg meat had moderate aroma. Ammonialike aroma, fishy aroma, grassy aroma, and fatty aroma were correlated with the gonads.

Keywords Eriocheir sinensis - GC-MS/O · Monolithic material sorptive extraction · Important odor compounds · Sensory evaluation

\section{Introduction}

Chinese mitten crab Eriocheir sinensis is a traditional savoury food in China, which originates from the coastal rivers and estuaries of the Yellow Sea. Currently, it has spread through Europe and California [1], and also constitutes a substantial freshwater fishery industry in China with the production increasing from 339,953 tons in 2002 to 714,380 tons in 2012 [2].

Many researchers [3-6] have previously revealed the discrepancies about constituents of flavor precursors (amino acids, fatty acids, nucleotides, etc.) among different edible parts of E. sinensis. Actually, the aroma profile of crab plays an important role for its purchase and consumption. In China, E. sinensis farmed in the Songjiang District in Shanghai is usually assumed to have high flavor quality. Besides, four edible portions of cooked E. sinensis - abdomen meat, claw meat, leg meat, and gonads - are individually popular, due to their unique pleasant aroma. The most common way of cooking the crab species is steaming. Therefore, four different edible parts of male E. sinensis farmed in Songjiang District were used as the raw materials in this study.

There exist hundreds of volatile compounds in crabmeat [7]. However, only a few of volatile compounds actively contribute to the aroma [8, 9]. Studies that used GC-MS to identify and quantify volatile compounds in crabs could not 
analyze the odor-active compounds in crab samples, but gas chromatography-olfactometry ( $\mathrm{GC}-\mathrm{O})$ provided a valuable tool for investigating the pattern of odorants, including the odor descriptors and activities [10]. By using GC-MS/O, Chen and Zhang [11] found trimethylamine, dimethyl sulfide, 1-octen-3-one, dimethyl trisulfide, 1-octen-3-ol, 3-(methythio)-propanal, benzaldehyde, and 2-acetylthiazole as odor-active compounds in crabmeat. Among these compounds, trimethylamine with fishy aroma and dimethyl sulfide with crabmeat aroma were detected to be important odorants, due to their high odor intensity in the $\mathrm{GC}-\mathrm{O}$ study. Yu and Chen [9] stated that aldehydes containing five carbon straight chains to nine carbon straight chains were important contributors to E. sinensis. Other researchers [12] detected 40 odorant compounds in four edible parts of $E$. sinensis. By combining the results of $\mathrm{GC}-\mathrm{O}$ analysis with odor activity values (OAVs), trimethylamine (fishy, ammonia-like odor), (Z)-4-heptenal (mushroom-like odor), and benzaldehyde (paint-like odor) were selected as important odorants in all of four edible parts of steamed male E. sinensis.

It could be easily found that most studies mentioned above only focused on the characterization of odor-active compounds in crabmeat. Almost none of them compared the aroma profiles of four edible parts with olfactometrically detected compounds. In this study, sensory evaluation, MMSE-GC-MS/O, and the E-Nose technique were applied to determine the aroma profiles of different edible parts of E. sinensis. Further, by PCA procedures, key aroma properties and important odor compounds for four edible parts of $E$. sinensis could be identified. Also, by a comparison of sensory evaluation and $\mathrm{GC}-\mathrm{O}$ detection, the relation between key aroma properties and specific odor compounds associated with certain edible part could be elucidated. This study may provide a theoretical basis for further mechanism research and development on IOCs in crabmeat or other aquatic products in the future.

\section{Materials and methods}

\section{Samples and reagents}

Twenty male E. sinensis of commercial size (average weight, ca. $200 \mathrm{~g}$ ) were harvested at the middle and latter part of October in 2013 from Songjiang (Shanghai), and immediately transported alive to the laboratory within $2 \mathrm{~h}$. Crabs were rinsed with tap water to remove foreign substances, and then cooked at a pot filled with distilled water and steamed for $30 \mathrm{~min}$. After cooling to room temperature, the crabmeat (including abdomen, claw and leg meat) and gonads of 20 male E. sinensis were separated and picked out manually. Each part of 20 male E. sinensis was mixed together, homogenized in an ice-bath condition (Model JZ-II, Tianjin Sifang Equipment Ltd, China), and then stored at $-80{ }^{\circ} \mathrm{C}$ until tested.

A standard solution of C5-C30 alkanes was used to calculate the linear retention indices (LRI) for each analyte, and an internal standard, 2,4,6-trimethylpyridine (TMP, purity $99 \%$ ), was used for quantification purposes. All the chemicals were purchased from Sigma-Aldrich (Shanghai, China).

\section{HS-MMSE procedure}

Volatile compounds from the samples were adsorbed using a previously described sampling kit [12]. Briefly, the sampling kit consists of seven monotrap ${ }^{\mathrm{TM}}(\mathrm{TM}) \mathrm{RCC} 18$ rods (external diameter $2.9 \mathrm{~mm}$; internal diameter $1 \mathrm{~mm}$; length $5 \mathrm{~mm}$; silica gel, activated carbon and octadecyl), the MT stand, and MT holder(type X5). Then $5 \mathrm{~g}$ of homogenized crab samples were fixed into a headspace vial $(15 \mathrm{ml})$. Then seven MT rods were placed at fixed positions in the headspace vial. This vial was kept in a water bath for $50 \mathrm{~min}$ at $100{ }^{\circ} \mathrm{C}$. After adsorption, the MT rods were removed and immediately placed in an adsorption tube and desorbed via a thermal desorption unit (TDU, Gerstel, Baltimore, MD, USA).

\section{Gas chromatography-mass spectrometry/olfactometry (GC-MS/O)}

The instrument used to select olfactometrically detected compounds from volatile compounds was a 7890 gas chromatography (GC) (Agilent Inc., Santa Clara) with 5975 mass selective detector (MSD) (CA, USA) and a sniffing port ODP-2 from Gerstel. The effluent from the capillary column was split $1: 1.5 \mathrm{v} / \mathrm{v}$ between the mass spectrometry detector (MSD). The capillary column used was a DB$5 \mathrm{MS}$ provided by Agilent (length $60 \mathrm{~m} \times$ internal diameter $0.32 \mathrm{~mm} \times$ film thickness $1 \mu \mathrm{m}$; Agilent Inc., USA). Helium was used as carrier gas at $1.2 \mathrm{ml} \mathrm{min}^{-1}$.

The oven temperature was programmed from 40 to $100{ }^{\circ} \mathrm{C}$ at $5{ }^{\circ} \mathrm{C} \mathrm{min}^{-1}$ with no initial hold, then to 180 at $2{ }^{\circ} \mathrm{C} \mathrm{min}{ }^{-1}$, to $250{ }^{\circ} \mathrm{C}$ at $5{ }^{\circ} \mathrm{C} \mathrm{min}{ }^{-1}$ with the final holds of $5 \mathrm{~min}$. MS conditions were as follows: detector interface temperature, $250{ }^{\circ} \mathrm{C}$; ion source temperature, $230{ }^{\circ} \mathrm{C}$; ionization energy, $70 \mathrm{eV}$; mass range, 40-450 amu; electron multiplier voltage, $1576 \mathrm{~V}$; and scan rate, $1.8 \mathrm{~s}^{-1}$. Samples were desorbed at $270{ }^{\circ} \mathrm{C}$ with a TDU (Gerstel) directly into the hot injector $\left(250{ }^{\circ} \mathrm{C}\right)$ of the gas chromatography with simultaneous cryofocusing with liquid nitrogen. 
A panel of five assessors ( 2 men and 3 women, aged from 22 to 35) from the laboratory staff with previous $\mathrm{GC}-\mathrm{O}$ sniffing experience was assembled in the $\mathrm{GC}-\mathrm{O}$ study. The assessors were asked to indicate the time, description, and odor intensity when an aroma was being detected. The scale used for intensity was $1-4(1=$ weak, $2=$ moderate, $3=$ strong, $4=$ very strong). The analysis followed the guidelines of Pollien, Ott, Montigon, Baumgartner, Munoz-Box and Chaintreau [13]. Each sample was evaluated once by each of the three assessors consecutively.

\section{Electronic nose (E-Nose) system}

Crab samples were further analyzed by a FOX4000 sensor array system (Alpha M.O.S., France) to distinguish the discrepancies of aroma profiles of four different edible parts. This detecting instrument consisted of an autosampling device, a detector unit containing 18 metal oxide sensors (MOS), and a pattern of recognition software for data recording and elaboration [7]. The total of 18 sensors could be divided into three classes and located in their own chambers: Sensor chamber CL: LY2/LG, LY2/G, LY2/AA, LY2/GH, LY2/gCTL, LY2/gCT; Sensor chamber A: T30/1,

Est. Conc. $(\mathrm{ng} / \mathrm{g})=\frac{\text { Peak area ratio }(\text { compound } / \mathrm{TMP}) \times 1 \mu \mathrm{g}(\mathrm{TMP})}{5 \mathrm{~g}(\text { hom ogenized crab sample })} \times 10^{3}$.
$\mathrm{P} 10 / 1, \mathrm{P} 10 / 2, \mathrm{P} 40 / 1, \mathrm{~T} 70 / 2, \mathrm{PA} / 2$; and Sensor chamber B: $\mathrm{P} 30 / 1, \mathrm{P} 40 / 2, \mathrm{P} 30 / 2, \mathrm{~T} 40 / 2, \mathrm{~T} 40 / 1, \mathrm{TA} / 2$.

Then $2 \mathrm{~g}$ of crab sample was loaded into a $10 \mathrm{ml}$ glass vessel, and the vessel was sealed by a metal screw cap immediately and placed in a specimen tray $\left(4{ }^{\circ} \mathrm{C}\right)$ for detection. The vessel was firstly balanced at $60{ }^{\circ} \mathrm{C}$ for $600 \mathrm{~s}$ before injection, and then $2500 \mu \mathrm{l}$ of the headspace gas was injected into the sensor chamber at $2500 \mu 1 / \mathrm{s}$ injection speed. Filtered and dried air (purity $>99.999 \%$ ), with a flow rate of $150 \mathrm{ml} / \mathrm{min}$, was used as a carrier gas for E-Nose detection. The data acquisition period lasted for $120 \mathrm{~s}$ and another $600 \mathrm{~s}$ was required for system rebalance. For each part, the E-Nose detection had been replicated five times under the same conditions.

\section{Sensory evaluation}

A panel of 8 people developed a consensus list of 5 terms (fishy, ammonia-like, fatty, grassy, and meat) to describe the aroma attributes of four edible parts of E. sinensis. They then used these terms to assess the aroma properties of the four different parts of samples, using a 10-point interval scale $(0=$ none, $9=$ extremely strong $)$. Sensory sessions took place in a sensory laboratory.

\section{Identification and quantification for volatile compounds}

Volatile compounds were identified by matching the mass spectra to the database spectra (Wiley/NIST 2008), and compared liner retention index (LRI) of each compound with its reference values. The LRI was calculated as follows:

$\mathrm{LRI}=\left(\frac{R t_{(x)}-R t_{(n)}}{R t_{(n+1)}-R t(n)}+n\right) \times 100$,

where $\operatorname{Rt}(x)$ is the retention time of each volatile compound $(x) ; \operatorname{Rt}(n)$ and $\operatorname{Rt}(n+1)$ are the retention times of $n$-alkanes eluting directly before and after the compound $(x)$ under identical chromatographic conditions.

Also, $1 \mu \mathrm{l}$ of TMP $1 \times 10^{2} \mathrm{ppm}$ in methanol was added to $5 \mathrm{~g}$ of homogenized sample as a chromatographic internal standard before the MMSE process. The calibration factors were all assumed to be 1.00 , and the estimated concentration of each volatile compound in the crab samples were calculated as follows:

\section{Statistical analysis}

Sensory evaluation and GC-MS/O detections were replicated three times in this study. The results of GC-MS were expressed as mean \pm standard deviation $(\mathrm{SD})(n=3)$, and the quantitative data for each compound were compared for the four edible parts of E. sinensis using variance (ANOVA). Principal component analysis (PCA) was performed based on the intensity of 39 odor compounds found by GC-MS/O using SPSS version 20.0 (SPSS Inc., Chicago, IL, USA).

\section{Results}

\section{Odor compounds detected by GC-MS/O}

The ODCs from four edible parts of E. sinensis are shown in Table 1, and the results of the quantitative analysis of the aroma compounds released by the four edible portions 
Table 1 Olfactometrically detected compounds in four edible parts of male Eriocheir sinensis farmed in Songjiang district by GC-MS/O detection

\begin{tabular}{|c|c|c|c|c|c|c|c|c|c|c|c|}
\hline \multirow[t]{2}{*}{ Number } & \multirow[t]{2}{*}{ LRI } & \multirow[t]{2}{*}{ Compounds } & \multirow[t]{2}{*}{ Odor description $^{\mathrm{a}}$} & \multicolumn{2}{|c|}{ Abdomen meat } & \multicolumn{2}{|c|}{ Claw meat } & \multicolumn{2}{|l|}{ Leg meat } & \multicolumn{2}{|l|}{ Gonad } \\
\hline & & & & Intensity $^{\mathrm{b}}$ & Identity $^{c}$ & Intensity & Identity & Intensity & Identity & Intensity & Identity \\
\hline 33 & 568 & Trimethylamine & Fishy & 2.4 & ODC & 3.4 & IOC & 3.6 & IOC & 3.4 & IOC \\
\hline 16 & 604 & 2-Butanone & Cheese & 1.2 & ODC & - & & 1 & ODC & 1.8 & ODC \\
\hline 1 & 663 & 3-Methylbutanal & Chocolate & 2.2 & ODC & 2.4 & & - & & 2.2 & ODC \\
\hline 2 & 671 & 2-Methylbutanal & Nutty & - & & 3.6 & IOC & - & & 1.4 & ODC \\
\hline 27 & 675 & Benzene & Special aroma & - & & 2.6 & ODC & 2.4 & ODC & 1.8 & ODC \\
\hline 24 & 686 & 1-Penten-3-ol & Mushroom & - & & - & & 3.8 & IOC & 4 & IOC \\
\hline 3 & 703 & Pentanal & Grassy & 2.4 & ODC & 1.8 & ODC & - & & - & \\
\hline 34 & 740 & Pyrazine & Nutty & 1.2 & ODC & 2 & ODC & 1 & ODC & - & \\
\hline 4 & 749 & 2-Methyl-2-butenal & Grassy & - & & 1 & ODC & 1.2 & ODC & - & \\
\hline 25 & 764 & 1-Pentanol & Fruit-like & 1.6 & UOC & - & & - & & - & \\
\hline 28 & 780 & Toluene & Special aroma & - & & 1 & ODC & 2.4 & ODC & - & \\
\hline 5 & 805 & Hexanal & Grassy & 1.8 & ODC & 1.8 & ODC & 2.4 & ODC & 3 & IOC \\
\hline 35 & 831 & Methylpyrazine & Popcorn & 1.8 & ODC & 1 & ODC & 2.4 & ODC & 1.2 & ODC \\
\hline 6 & 867 & 2-n-Butylacrolein & Floral & 1.2 & ODC & - & & 1.2 & ODC & - & \\
\hline 17 & 892 & 2-Heptanone & Cheese & 1 & ODC & 2.6 & ODC & 1 & ODC & 2.4 & ODC \\
\hline 7 & 903 & (Z)-4-Heptenal & Fishy & 2.2 & ODC & 2.4 & ODC & 4 & IOC & 2.4 & ODC \\
\hline 8 & 905 & Heptanal & Fatty & 2.8 & ODC & 2.6 & ODC & 2.8 & ODC & 3 & IOC \\
\hline 36 & 912 & 2-Ethylpyridine & Roast potato & 3 & IOC & 3.4 & IOC & 3 & IOC & 3 & IOC \\
\hline 37 & 919 & 2,5-Dimethylpyrazine & Popcorn & 2 & ODC & 3.4 & IOC & 2.4 & ODC & 3.2 & IOC \\
\hline 38 & 925 & 2,3-Dimethylpyrazine & Popcorn & 2 & ODC & 3 & IOC & - & & 3 & IOC \\
\hline 9 & 977 & Benzaldehyde & Almond & 2.2 & ODC & 3 & IOC & 3 & IOC & 3 & IOC \\
\hline 26 & 979 & 1-Octen-3-ol & Mushroom & 1.6 & ODC & 3 & IOC & 2 & ODC & 2 & ODC \\
\hline 18 & 985 & $\begin{array}{l}\text { 6-Methyl-5-hepten- } \\
\text { 2-one }\end{array}$ & Sour plum & 2.2 & ODC & 2.4 & ODC & 2.8 & ODC & - & \\
\hline 19 & 991 & 2-Octanone & Fruit-like & - & & - & & - & & 2.4 & UOC \\
\hline 31 & 995 & 2-Pentylfuran & Sweet,green & 1.2 & ODC & 1.8 & ODC & 2.4 & ODC & 1.6 & ODC \\
\hline 10 & 1006 & Octanal & Orange & - & & 1.2 & ODC & 1.8 & ODC & - & \\
\hline 39 & 1008 & Trimethylpyrazine & Chocolate & 2.4 & ODC & 1.8 & ODC & 2.4 & ODC & - & \\
\hline 32 & 1029 & 2-Acetylthiazole & Roast meat & - & & - & & - & & 3 & $\begin{array}{r}\text { IOC \& } \\
\text { UOC }\end{array}$ \\
\hline 11 & 1056 & Benzeneacetaldehyde & Floral & - & & 2.4 & UOC & - & & - & \\
\hline 20 & 1091 & 2-Nonanone & Fruit-like & - & & 2.4 & ODC & 3 & IOC & 1.8 & ODC \\
\hline 21 & 1098 & $\begin{array}{l}\text { (E,E)-3,5-Octadien- } \\
\text { 2-one }\end{array}$ & Sweet, milk & 1.2 & ODC & 1.2 & ODC & 2.4 & ODC & 2.2 & ODC \\
\hline 12 & 1101 & $\begin{array}{l}\text { 3-Methyl-2-thiophene- } \\
\text { carboxaldehyde }\end{array}$ & Chocolate & 3.4 & IOC \& UOC & - & & - & & - & \\
\hline 13 & 1107 & Nonanal & Green & - & & 2.2 & ODC & 2 & ODC & 2.8 & ODC \\
\hline 14 & 1179 & 4-Ethylbenzaldehyde & Almond & 1.2 & ODC & 2.4 & ODC & 2.4 & ODC & 3.2 & IOC \\
\hline 22 & 1192 & 2-Decanone & Fruit-like & 1 & ODC & 1 & ODC & 3 & IOCs & - & \\
\hline 15 & 1209 & Decanal & Orange & - & & 1.2 & UOC & - & & - & \\
\hline 29 & 1214 & Naphthalene & Camphor ball & 2.4 & UOC & - & & - & & - & \\
\hline 23 & 1293 & 2-Undecanone & Fruit-like & - & & - & & 2.4 & ODC & 1.8 & ODC \\
\hline 30 & 1344 & 2-Methylnaphthalene & Plastic & - & & 2.2 & UOC & - & & - & \\
\hline
\end{tabular}

a Odor description described by three experienced assessors during $\mathrm{GC}-\mathrm{O}$ detection

${ }^{\mathrm{b}}$ Odor intensity reported by three experienced assessors during GC-O detection $(1=$ weak intensity, $2=$ moderate intensity, $3=$ strong intensity, and 4 = very strong intensity)

${ }^{c}$ Identity of odorants were due to their odor intensities. $O D C$ olfactometrically detected compounds (odor intensity value $>1$ ), IOC important odor compounds (odor intensity value $\geq 3$ ), $U O C$ unique odor compounds only existed in one part

—, data not detected or available

- Olfactometrically detected compounds found to be common in all of four edible parts 
Table 2 Estimated concentrations of olfactometrically detected compounds identified in different edible parts of male Eriocheir sinensis by MMSE-GC-MS $\left(\mu \mathrm{g} \mathrm{kg}^{-1}, n=3\right)$

\begin{tabular}{|c|c|c|c|c|c|c|c|}
\hline Number & $\mathrm{LRI}^{\mathrm{e}}$ & Compounds & Identification $^{\mathrm{f}}$ & Abdomen meat & Claw meat & Leg meat & Gonad \\
\hline \multicolumn{8}{|c|}{ Aldehydes (15) } \\
\hline 1 & 663 & 3-Methylbutanal & MS, LRI & $30.4 \pm 1.4 \mathrm{~b}$ & $38.8 \pm 2.2 \mathrm{a}$ & $12.1 \pm 0.8 \mathrm{c}$ & $30.5 \pm 1.7 \mathrm{~b}$ \\
\hline 2 & 671 & 2-Methylbutanal & MS, LRI & $28.2 \pm 1.9 \mathrm{c}$ & $98.1 \pm 4.7 \mathrm{a}$ & N.D. d & $57.2 \pm 3.0 \mathrm{~b}$ \\
\hline 3 & 703 & Pentanal & MS, LRI & $45.8 \pm 3.0 \mathrm{c}$ & $13.7 \pm 1.2 \mathrm{a}$ & $8.6 \pm 0.3 b$ & $11.8 \pm 0.8 \mathrm{~b}$ \\
\hline 4 & 749 & 2-Methyl-2-butenal & MS, LRI & $13.6 \pm 0.6 \mathrm{c}$ & $28.6 \pm 1.4 \mathrm{~b}$ & $42.1 \pm 2.2 \mathrm{a}$ & $16.0 \pm 0.8 \mathrm{c}$ \\
\hline 5 & 805 & Hexanal & MS, LRI & $67.1 \pm 5.8 \mathrm{c}$ & $52.6 \pm 3.4 \mathrm{~d}$ & $86.1 \pm 4.8 b$ & $173.6 \pm 12.3 \mathrm{a}$ \\
\hline 6 & 867 & 2-n-Butylacrolein & MS, LRI & $12.8 \pm 0.6 \mathrm{c}$ & $5.6 \pm 0.5 \mathrm{~d}$ & $14.8 \pm 0.8 b$ & N.D. a \\
\hline 7 & 903 & (Z)-4-Heptenal & MS, LRI & $16.9 \pm 1.0 \mathrm{~b}$ & $20.7 \pm 1.6 b$ & $50.7 \pm 3.5 \mathrm{a}$ & $23.3 \pm 1.6 \mathrm{~b}$ \\
\hline 8 & 905 & Heptanal & MS, LRI & $28.9 \pm 2.4 \mathrm{c}$ & $25.2 \pm 0.9 \mathrm{c}$ & $40.1 \pm 1.4 \mathrm{~b}$ & $60.9 \pm 3.4 \mathrm{a}$ \\
\hline 9 & 977 & Benzaldehyde & MS, LRI & $408.7 \pm 18.3 \mathrm{~b}$ & $468.9 \pm 38.6 b$ & $577.6 \pm 34.8 \mathrm{a}$ & $617.4 \pm 37.5 \mathrm{a}$ \\
\hline 10 & 1006 & Octanal & MS, LRI & $16.5 \pm 0.8 b$ & $16.9 \pm 1.1 \mathrm{c}$ & $29.8 \pm 2.6 \mathrm{~b}$ & $34.9 \pm 2.3 \mathrm{a}$ \\
\hline 11 & 1056 & Benzeneacetaldehyde & MS, LRI & N.D. ${ }^{g} \mathrm{~b}$ & $4.2 \pm 0.3 \mathrm{a}$ & N.D. b & N.D. b \\
\hline 12 & 1101 & $\begin{array}{l}\text { 3-Methyl-2-thiophenecarboxalde- } \\
\text { hyde }\end{array}$ & MS & $33.4 \pm 1.5 \mathrm{a}$ & $5.7 \pm 0.5 b$ & N.D. c & N.D. c \\
\hline 13 & 1107 & Nonanal & MS, LRI & $28.9 \pm 2.5 \mathrm{c}$ & $38.1 \pm 2.4 \mathrm{~b}$ & $29.9 \pm 2.2 \mathrm{c}$ & $91.7 \pm 6.9 \mathrm{a}$ \\
\hline 14 & 1179 & 4-Ethylbenzaldehyde & MS, LRI & $18.6 \pm 1.0 \mathrm{~b}$ & $22.0 \pm 0.8 \mathrm{~b}$ & $19.5 \pm 1.6 \mathrm{~b}$ & $51.6 \pm 3.0 \mathrm{a}$ \\
\hline \multirow[t]{2}{*}{15} & 1209 & Decanal & MS, LRI & $6.9 \pm 0.5 b$ & $12.5 \pm 0.6 \mathrm{a}$ & $5.6 \pm 0.3 \mathrm{c}$ & $12.0 \pm 0.7 \mathrm{a}$ \\
\hline & & Subtotal & & $756.7 \pm 41.4 \mathrm{~b}$ & $812.5 \pm 60.0 \mathrm{~b}$ & $916.7 \pm 55.8 \mathrm{~b}$ & $1180.8 \pm 73.9 \mathrm{a}$ \\
\hline \multicolumn{8}{|c|}{ Ketones (5) } \\
\hline 16 & 604 & 2-Butanone & MS, LRI & $26.7 \pm 2.2 b$ & $18.6 \pm 1.6 \mathrm{c}$ & N.D.d & $38.6 \pm 3.1 \mathrm{a}$ \\
\hline 17 & 892 & 2-Heptanone & MS, LRI & $20.3 \pm 1.5 \mathrm{c}$ & $53.1 \pm 3.1 \mathrm{a}$ & $21.5 \pm 0.8 \mathrm{c}$ & $30.3 \pm 1.8 b$ \\
\hline 18 & 985 & 6-Methyl-5-hepten-2-one & MS, LRI & $14.5 \pm 0.6 b$ & $15.5 \pm 0.7 \mathrm{~b}$ & $35.4 \pm 1.7 \mathrm{a}$ & $11.0 \pm 0.5 \mathrm{c}$ \\
\hline 19 & 991 & 2-Octanone & MS, LRI & $13.0 \pm 0.9 \mathrm{~b}$ & $14.9 \pm 1.1 \mathrm{~b}$ & $25.1 \pm 2.2 \mathrm{a}$ & $23.1 \pm 1.8 \mathrm{a}$ \\
\hline 20 & 1091 & 2-Nonanone & MS, LRI & $17.2 \pm 0.6 \mathrm{c}$ & $19.2 \pm 1.3 \mathrm{c}$ & $42.3 \pm 3.0 \mathrm{a}$ & $27.5 \pm 1.5 \mathrm{~b}$ \\
\hline 21 & 1098 & (E,E)-3,5-Octadien-2-one & MS, LRI & $22.6 \pm 0.9 \mathrm{~d}$ & $26.6 \pm 2.2 \mathrm{c}$ & $43.9 \pm 1.3 b$ & $63.8 \pm 3.2 \mathrm{a}$ \\
\hline 22 & 1192 & 2-Decanone & MS, LRI & $20.0 \pm 1.5 b$ & $19.0 \pm 1.6 \mathrm{~b}$ & $51.4 \pm 4.1 \mathrm{a}$ & $17.6 \pm 1.1 \mathrm{~b}$ \\
\hline \multirow[t]{2}{*}{23} & 1293 & 2-Undecanone & MS & $14.0 \pm 1.1 \mathrm{~b}$ & $15.6 \pm 1.2 \mathrm{~b}$ & $22.5 \pm 1.6 \mathrm{a}$ & $21.2 \pm 1.6 \mathrm{a}$ \\
\hline & & Subtotal & & $148.2 \pm 9.2 \mathrm{c}$ & $182.5 \pm 12.9 \mathrm{~b}$ & $242.0 \pm 14.7 \mathrm{a}$ & $233.1 \pm 14.5 \mathrm{a}$ \\
\hline \multicolumn{8}{|c|}{ Alcohols (3) } \\
\hline 24 & 686 & 1-Penten-3-ol & MS, LRI & $112.0 \pm 8.8 \mathrm{c}$ & N.D. d & $207.9 \pm 15.9 b$ & $241.1 \pm 15.7 \mathrm{a}$ \\
\hline 25 & 764 & 1-Pentanol & MS, LRI & $41.6 \pm 2.9 \mathrm{a}$ & $8.6 \pm 0.6 \mathrm{~d}$ & $14.0 \pm 1.0 \mathrm{c}$ & $28.0 \pm 2.0 \mathrm{~b}$ \\
\hline \multirow[t]{2}{*}{26} & 979 & 1-Octen-3-ol & MS, LRI & $89.3 \pm 4.2 \mathrm{c}$ & $170.4 \pm 13.7 \mathrm{a}$ & $87.8 \pm 3.5 \mathrm{c}$ & $118.1 \pm 6.5 \mathrm{~b}$ \\
\hline & & Subtotal & & $242.9 \pm 15.9 \mathrm{~d}$ & $179.0 \pm 14.3 \mathrm{c}$ & $309.7 \pm 20.4 \mathrm{~b}$ & $386.2 \pm 24.2 \mathrm{a}$ \\
\hline \multicolumn{8}{|c|}{ Aromatics (4) } \\
\hline 27 & 675 & Benzene & MS, LRI & N.D. b & $106.4 \pm 5.4 \mathrm{a}$ & $100.7 \pm 6.2 \mathrm{a}$ & $92.8 \pm 7.4 \mathrm{a}$ \\
\hline 28 & 780 & Toluene & MS, LRI & $9.2 \pm 0.3 \mathrm{a}$ & $10.3 \pm 0.6 \mathrm{a}$ & $11.1 \pm 0.8 \mathrm{a}$ & $10.2 \pm 0.7 \mathrm{a}$ \\
\hline 29 & 1214 & Naphthalene & MS, LRI & $24.7 \pm 1.7 \mathrm{a}$ & $19.4 \pm 1.7 \mathrm{~b}$ & $14.3 \pm 1.1 \mathrm{c}$ & $13.9 \pm 1.0 \mathrm{c}$ \\
\hline \multirow[t]{2}{*}{30} & 1344 & 2-Methylnaphthalene & MS & $24.9 \pm 2.1 \mathrm{~b}$ & $30.3 \pm 1.8 \mathrm{a}$ & $14.9 \pm 0.7 \mathrm{c}$ & $22.1 \pm 1.4 \mathrm{~b}$ \\
\hline & & Subtotal & & $58.7 \pm 4.1 \mathrm{c}$ & $166.3 \pm 9.5 b$ & $141.0 \pm 8.8 \mathrm{a}$ & $139.0 \pm 10.6 \mathrm{a}$ \\
\hline \multicolumn{8}{|c|}{ Furans (1) } \\
\hline \multirow[t]{2}{*}{31} & 995 & 2-Pentylfuran & MS, LRI & $105.0 \pm 4.0 \mathrm{c}$ & $106.8 \pm 3.4 \mathrm{c}$ & $212.2 \pm 18.1 \mathrm{a}$ & $128.5 \pm 6.4 \mathrm{~b}$ \\
\hline & & Subtotal & & $105.0 \pm 4.0 \mathrm{c}$ & $106.8 \pm 3.4 \mathrm{c}$ & $212.2 \pm 18.1 \mathrm{a}$ & $128.5 \pm 6.4 \mathrm{~b}$ \\
\hline \multicolumn{8}{|c|}{ S-containing (1) } \\
\hline \multirow[t]{2}{*}{32} & 1029 & 2-Acetylthiazole & MS, LRI & $5.5 \pm 0.4 \mathrm{~b}$ & $4.8 \pm 0.3 b$ & $2.7 \pm 0.2 \mathrm{c}$ & $17.1 \pm 1.1 \mathrm{a}$ \\
\hline & & Subtotal & & $5.5 \pm 0.4 \mathrm{~b}$ & $4.8 \pm 0.3 b$ & $2.7 \pm 0.2 \mathrm{c}$ & $17.1 \pm 1.1 \mathrm{a}$ \\
\hline \multicolumn{8}{|c|}{ N-containing (7) } \\
\hline 33 & 568 & Trimethylamine & MS, LRI & $853.2 \pm 44.9 \mathrm{~b}$ & $690.5 \pm 42.1 \mathrm{c}$ & $2954.0 \pm 146.0 \mathrm{a}$ & $517.8 \pm 43.3 \mathrm{~d}$ \\
\hline 34 & 740 & Pyrazine & MS, LRI & $34.9 \pm 1.7 \mathrm{a}$ & $36.9 \pm 1.5 \mathrm{a}$ & $20.4 \pm 1.1 \mathrm{~b}$ & N.D. c \\
\hline
\end{tabular}


Table 2 continued

\begin{tabular}{|c|c|c|c|c|c|c|c|}
\hline Number & $\mathrm{LRI}^{\mathrm{e}}$ & Compounds & Identification $^{\mathrm{f}}$ & Abdomen meat & Claw meat & Leg meat & Gonad \\
\hline 35 & 831 & Methylpyrazine & MS, LRI & $51.4 \pm 2.3 \mathrm{a}$ & $32.8 \pm 2.1 \mathrm{~b}$ & $47.4 \pm 2.6 \mathrm{a}$ & $36.6 \pm 2.0 \mathrm{~b}$ \\
\hline 36 & 912 & 2-Ethylpyridine & MS, LRI & $13.4 \pm 0.8 \mathrm{~d}$ & $19.4 \pm 1.7 \mathrm{c}$ & $33.8 \pm 1.2 \mathrm{a}$ & $26.6 \pm 1.6 \mathrm{~b}$ \\
\hline 37 & 919 & 2,5-Dimethylpyrazine & MS, LRI & $35.8 \pm 3.1 \mathrm{~b}$ & $55.7 \pm 2.0 \mathrm{a}$ & $31.8 \pm 2.1 \mathrm{~b}$ & $56.0 \pm 3.7 \mathrm{a}$ \\
\hline 38 & 925 & 2,3-Dimethylpyrazine & MS & $9.7 \pm 0.5 \mathrm{~b}$ & $12.5 \pm 0.5 \mathrm{a}$ & N.D. c & $10.2 \pm 0.5 b$ \\
\hline \multirow[t]{3}{*}{39} & 1008 & Trimethylpyrazine & MS, LRI & $30.6 \pm 2.3 b$ & $45.3 \pm 2.4 \mathrm{a}$ & $48.5 \pm 3.0 \mathrm{a}$ & $41.2 \pm 2.3 \mathrm{a}$ \\
\hline & & Subtotal & & $1029.0 \pm 55.6 \mathrm{c}$ & $893.1 \pm 52.3 \mathrm{~d}$ & $3135.9 \pm 156.0 \mathrm{a}$ & $688.3 \pm 53.6 b$ \\
\hline & & Total & & $2346.0 \pm 126.2 \mathrm{c}$ & $2345.0 \pm 152.0 \mathrm{c}$ & $4960.2 \pm 273.8 \mathrm{a}$ & $2773.0 \pm 184.2 \mathrm{~b}$ \\
\hline
\end{tabular}

Means in the same row followed by different letters are statistically different $(P<0.05)$

e Linear retention index (LRI) on a DB-5MS column

${ }^{\mathrm{f}}$ Means of identification: $M S$ mass spectra (identified from the mass spectra deposited in a database), LRI linear retention index (compared with the LRI in the literature)

g N.D. not detected

Fig. 1 Principal component plot (PC1 versus PC2) of four edible parts, showing correlations with aroma volatiles (Numbers on plot refer to compound numbers in Table 1)

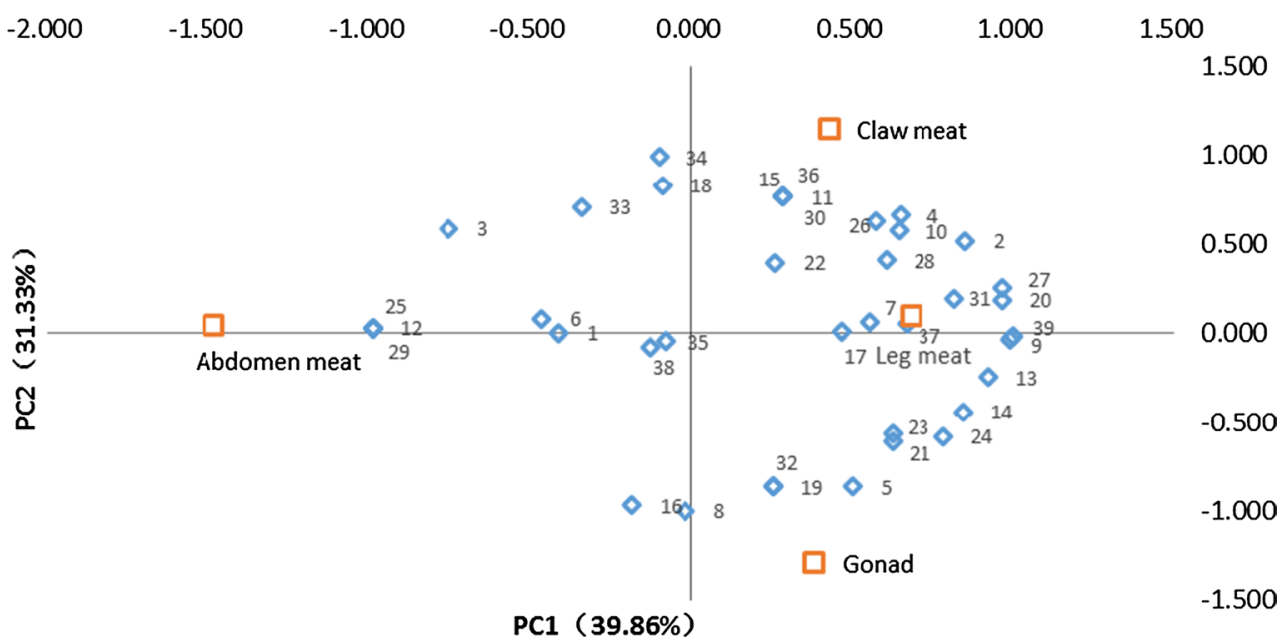

are shown in Table 2. Thirty-nine ODCs were identified. Within all the ODCs, 26 compounds were found in abdomen meat, 30 were detected in claw meat, 27 were perceived in leg meat, and 24 were found in the gonad. Only 13 ODCs were detected to be common to all four parts. ODCs with an odor intensity no less than three could be determined as important odor compounds (IOCs), these compounds may be regarded as major contributors to the aroma profile of different edible parts. 2-Ethylpyridine (roast potato aroma) and 3-methyl-2-thiophenecarboxaldehyde (chocolate aroma) were found as IOCs in abdomen meat. Seven IOCs were detected in claw meat, they were trimethylamine (fishy aroma), 2-methylbutanal (nutty aroma), 2-ethylpyridine (roast potato aroma), 2,5-dimethylpyrazine (popcorn aroma), 2,3-dimethylpyrazine (popcorn aroma), benzaldehyde (almond aroma) and 1-octen-3-ol (mushroom aroma). Seven IOCs were found in leg meat, they were trimethylamine (fishy aroma), 1-penten-3-ol (mushroom aroma), 2-ethylpyridine (roast potato aroma), benzaldehyde (almond aroma), 2-nonanone (fruit-like aroma), and 2-decanone (fruit- like aroma). Ten IOCstrimethylamine (fishy aroma), 1-penten-3-ol (mushroom aroma), hexanal (grassy aroma), heptanal (fatty aroma), 2-ethylpyridine (roast potato aroma), 2,5-dimethylpyrazine (popcorn aroma), 2,3-dimethylpyrazine (popcorn aroma), benzaldehyde (almond aroma), 2-acetylthiazole (roast meat aroma), and 4-ethylbenzaldehyde (almond aroma)—were detected in gonad parts.

ODCs that were only perceived in one edible part were determined to be unique odor compounds (UOCs). Three UOCs, 3-methyl-2-thiophenecarboxaldehyde (chocolate aroma), 1-pentanol (fruit-like aroma), and naphthalene (camphor ball aroma), were detected only in abdomen meat. Benzeneacetaldehyde (floral aroma), decanal (orange aroma), and 2-methylnaphthalene (plastic aroma) were only perceived in claw meat. 2-Octanone (fruit-like aroma) and 2-acetylthiazole (roast meat) were only found in the gonad. 
Fig. 2 PCA analyses of four edible parts of $E$. sinensis from E-Nose response data

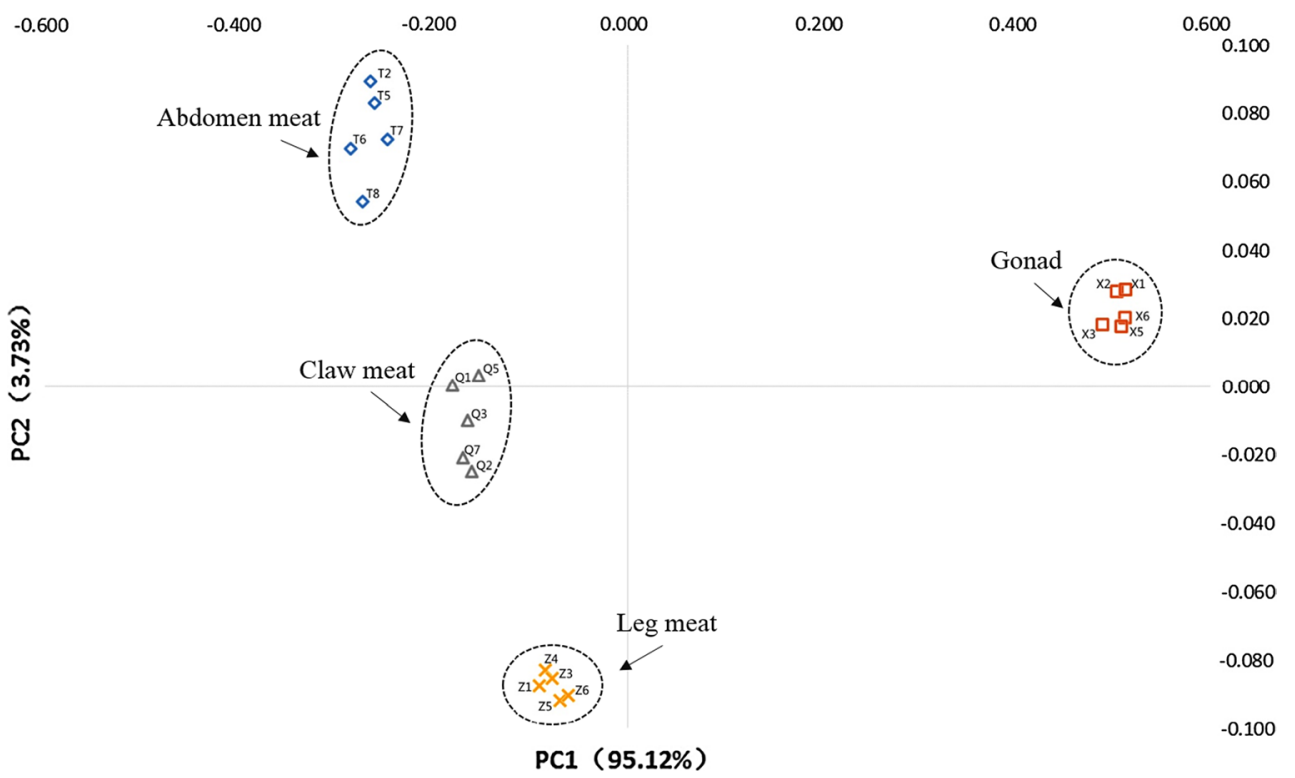

Fig. 3 Graph of the mean sensory scores of four edible parts of Chinese mitten crab

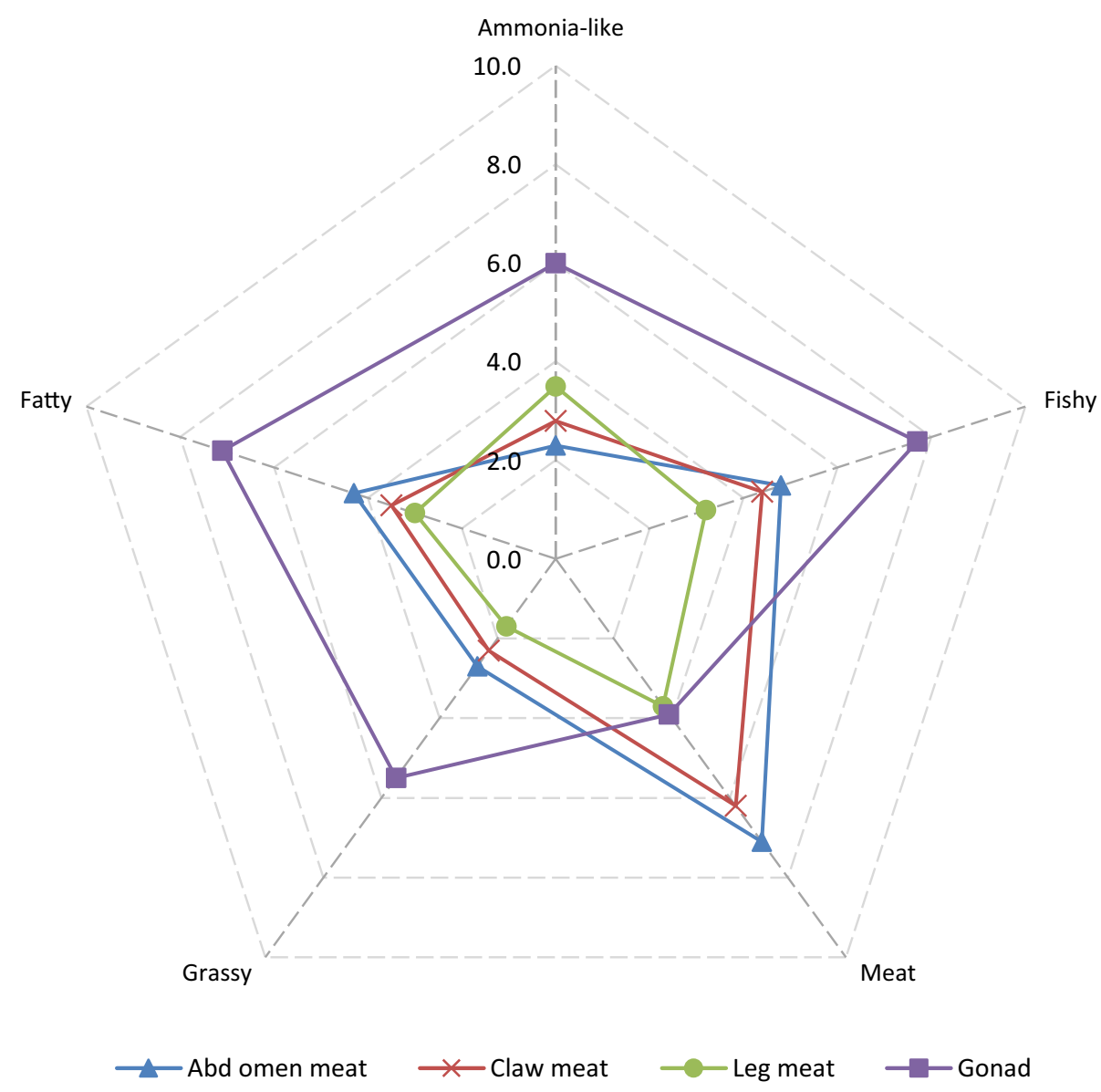

(Fig. 1). Two principal components accounted for more than $70 \%$ of the variation in the data: principal component 1 (PC 1) displayed $39.86 \%$ of the variation and principal component 2 (PC 2) displayed $31.33 \%$. Abdomen meat
Principal component analysis was carried out to visualize the differences between the four edible parts of E. sinensis 
Fig. 4 Principal component plot (PC1 versus PC2) of four edible parts, showing correlations with sensory attributes

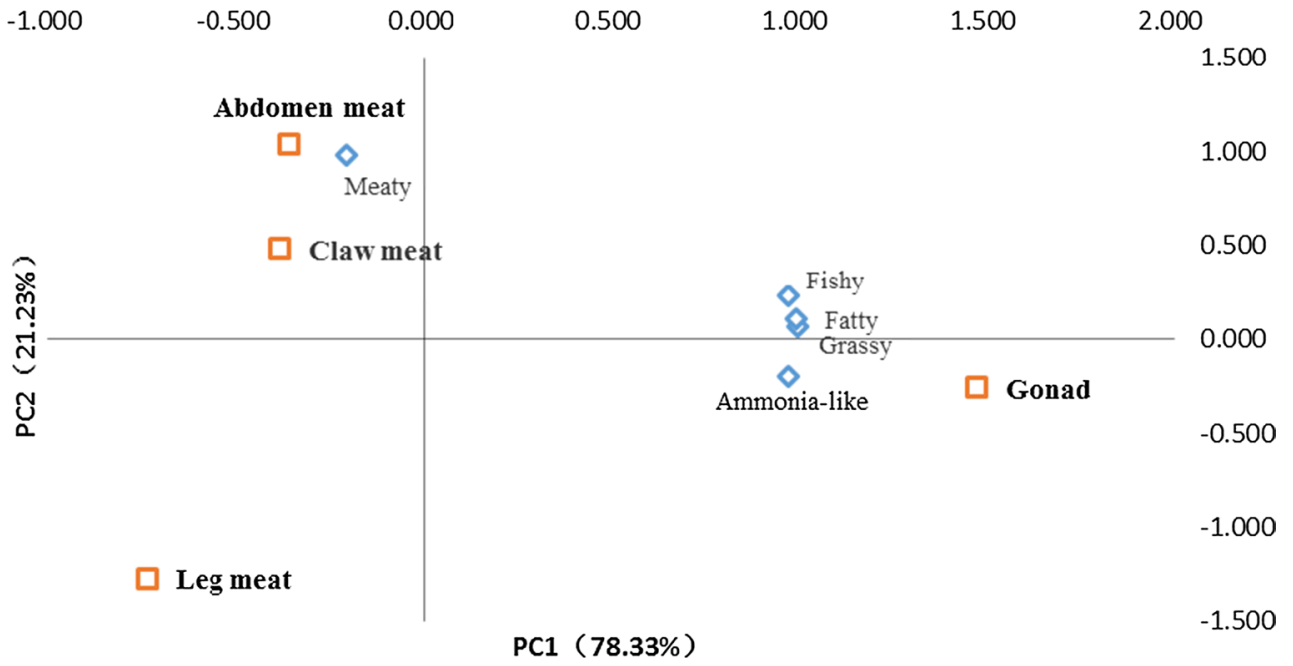

was separated from the other three parts across PC1. The volatile compounds correlated with abdomen meat included 3-methyl-2-thiophenecarboxaldehyde, 1-pentanol, and naphthalene. The gonad was separated from the other three parts across PC2. Hexanal, 2-octanone and 2-acetylthiazole were associated with the gonad. In addition, benzeneacetaldehyde, decanal, 2-methylnaphthalene, and 2-ethylpyridine were correlated with claw meat, while 2,5-dimethylpyrazine, 2-pentylfuran (floral aroma) and (Z)-4-heptenal were associated with leg meat.

\section{Electronic nose response to the aroma of four edible parts of $E$. sinensis}

To elaborate the discrepancies on aroma profiles of four edible parts of E. sinensis, an E-Nose experiment was performed in this study, and the PCA procedure was applied for data processing. As shown in Fig. 2, PC 1 and PC2 explained 95.12 and $3.73 \%$ of sample variation, respectively. From Fig. 2, sample dots of four edible parts could be distinguished well. Sample dots of three kinds of crabmeat (abdomen, claw and leg meat) were all located in the second quadrant and third quadrant and found relatively close to each other, while sample dots of the gonad part that was located in the first quadrant were well separated from those of the crabmeat across PC 1, indicating that major differences could be found between aroma profiles of the gonad part and three kinds of crabmeat.

\section{Sensory analysis of four edible parts}

Five aroma attributes were used to describe the sensory properties of four edible parts of Chinese mitten crab. The aromas were described as fishy, ammonia-like, fatty, grassy, and meat.
As shown in Fig. 3, abdomen meat scored highest for meat aroma, as well as scoring higher than claw meat and leg meat for fishy aroma, grassy aroma and fatty aroma. Meat aroma was higher in claw meat than leg meat and the gonad, as well as fishy aroma, grassy aroma and fatty aroma were higher in claw meat than leg meat. Ammonialike aroma was higher in leg meat than abdomen meat and claw meat. Ammonia-like aroma, fishy aroma, grassy aroma, and fatty aroma were all higher in the gonad than the other three parts.

\section{Principal component analysis (PCA) of sensory evaluation}

Principal component analysis was also carried out on the correlation matrix of all samples and all attributes (Fig. 4). Again, all of the variation in the data was explained by two principal components. PC 1 displayed $78.33 \%$ of the variation and PC 2 displayed $21.23 \%$. The gonad was separated from the other three parts across PC1. The attributes associated with the gonad were fishy aroma, fatty aroma, grassy aroma, and ammonia-like aroma. Whereas, meaty aroma was correlated with abdomen meat and claw meat.

\section{Discussion}

According to the data of GC-MS/O (Tables 1, 2), 6 IOCs were found in no less than two edible parts of $E$. sinensis. Among them, 2-ethylpyridine was the only IOC found in all four edible parts in this study (Table 1). It has been reported in krill oil [14] and dried Acetes chinensis [15], and also demonstrated as important odorants in claw meat, leg meat, and the gonad of $E$. sinensis, but not found in abdomen meat [12]. The origin of 2-ethylpyridine is likely to be decompositions of DHA and EPA upon cooking 
[16]. Guo et al. [17] have reported relatively high levels of C20:5n-3 and C22:6n-3 in all four edible parts of E. sinensis (abdomen meat: 10.99 and $9.49 \%$; claw meat: 12.97 and $8.33 \%$; leg meat: 15.89 and $11.18 \%$; gonad: 4.39$3.59 \%$ ), which may explain the high intensity of 2-ethylpyridine in our present study.

Trimethylamine and benzaldehyde presented high intensity scores in claw meat, leg meat, and the gonad respectively. Trimethylamine is a thermal decomposition product of choline, betaine, methionine, or most likely, trimethylamine oxide during cooking [18]. It has been reported as an important odorant in cooked E. sinensis [11], lobster [18], and cod [19] in high concentrations. Due to its high content and intense aroma in this study, trimethylamine could be a net contributor to the aroma of three parts-claw meat, leg meat, and the gonad-of E. sinensis. Benzaldehyde was also found with high intensities in claw meat, leg meat and gonad, but not found as the IOC in abdomen meat. The quantitative analysis (Table 2) did report a lower quantity of benzaldehyde in abdomen meat. It has been reported as important compounds in E. sinensis with paint-like aroma [12], and has been also reported as key odor-active compounds in steamed Coilia ectenes meat, with a "bitter almond" descriptor [20]. It is not surprising that benzaldehyde was found to be important as it is considered to be one of the lipid oxidation products with high aromatic impact [21].

2,5-Dimethylpyrazine only presented a strong popcorn aroma in claw meat and the gonad. The estimated concentrations of 2,5-dimethylpyrazine in claw meat and the gonad were $55.7 \pm 2.0$ and $56.0 \pm 3.7 \mu \mathrm{g} \mathrm{kg}^{-1}$ (Table 2), respectively, much higher than that of in abdomen meat $\left(35.8 \pm 3.1 \mu \mathrm{g} \mathrm{kg}^{-1}\right)$ and leg meat $\left(31.8 \pm 2.1 \mu \mathrm{g} \mathrm{kg}^{-1}\right)$. Another N-containing compound, 2,3-dimethylpyrazine, has also been detected to contribute a strong popcorn aroma to the claw meat and gonad of E. sinensis. It has not been reported as odor-active components in E. sinensis before, but has already been described with popcorn and rice aroma in salted-dried white herring [22]. These two compounds are probably thermally generated via Maillard and pyrolysis reactions through Strecker degradations from various nitrogen sources such as amino acids in heat-processed foods [15, 23].

1-Penten-3-ol was identified as IOC in both leg meat and the gonad of $E$. sinensis. It has been detected as a high intensity odorant only in the gonad of $E$. sinensis farmed in Yangcheng Lake. The estimated concentration of 1-penten3-ol was highest in the gonad $\left(241.1 \pm 15.7 \mu \mathrm{g} \mathrm{kg}^{-1}\right)$, followed by leg meat $\left(207.9 \pm 15.9 \mu \mathrm{g} \mathrm{kg}^{-1}\right)$ and abdomen meat $\left(112.0 \pm 8.8 \mu \mathrm{g} \mathrm{kg}^{-1}\right)$, and it was not found in claw meat. This alcohol is generated from PUFAs oxidation catalyzed by lipoxygenase or hydroperoxidase [20]. It is not surprising that the intensity of 1-penten-3-ol was high in leg meat, as its precursor - PUFAs - has been detected at a relatively high level of the leg meat of E. sinensis [17].

Differences have also been detected in four edible portions (Figs. 2, 3, 4). The gonad part contained the largest amount of IOCs, and possessed the strongest aroma in ammonia-like, fishy, grassy, and fatty attributes in sensory analysis. This might result from the flavor precursor (amino acids, fatty acids, etc.) discrepancies between the gonad and the other three parts. The gonad was rich in fatty acid, while crabmeats contained more protein. Crude fat content in the gonad of E. sinensis was 23.53-26.30\%, much higher than that in the crabmeat (0.44-1.29\%) [17]. $N-3$ polyunsaturated fatty acids (PUFA) were revealed as important precursors in $E$. sinensis, although the percentage of $n-3$ PUFA in total fatty acids (TFA) in the gonad was $14.5-15.1 \%$, a little lower than that in the crabmeat (15.60-24.88\%) [24]; the content of $n-3$ PUFA in E. sinensis's gonad was still higher than that in crabmeat [25]. Hexanal, 2-octanone, and 2-acetylthiazole were closely related to the gonad part (Fig. 1). Among them, hexanal has been widely reported in crab species [5, $7,12]$. It is usually considered as the degradation product of linoleate and linolenate hydroperoxides [7, 26]. The origin of 2-octanone is likely to be a thermal degradation of PUFAs [27]. 2-Acetylthiazole was the UOC identified in the gonad with a high intensity score. It has already been reported in E. sinensis [7] and lobster [18] in similar concentrations. The origin of 2-acetylthiazole in the sample is probably a thermal degradation of cysteine [18].

Sample dots of abdomen meat and claw meat in Fig. 4 were both located in the second quadrant, and the meaty aroma attribute was closely related to these two parts. The aroma of abdomen meat was found to be correlated with 3-methyl-2-thiophenecarboxaldehyde, 1-pentanol, and naphthalene. Benzeneacetaldehyde, decanal, 2-ethylpyridine, and 2-methylnaphthalene were correlated with claw meat (Fig. 1). Unlike the high levels of fatty acids in the gonad, abdomen meat and claw meat of $E$. sinensis are rich in amino acids [17]. The branched chain aldehydes as well as phenyl-containing aldehydes are aroma components usually generated from the Strecker reaction of amino acids: e.g., 3-methyl-2-thiophenecarboxaldehyde (sulphur amino acids) [28] and benzeneacetaldehyde (phenylalamine) [7]. Among them, 3-Methyl-2-thiophenecarboxaldehyde is worth mentioning because of its high odor intensity in abdomen meat. In addition, decanal is the degradation product of linoleate and linolenate hydroperoxides [7, 26]. 1-Pentanol is probably formed from branched-chain amino acid precursors [29]. 1-Octen-3-ol is considered as a degradation product of linoleic acid hydroperoxides [20]. These compounds have been previously reported as odor-active compounds in crabs species [12] and other aquatic foods, such as smoked salmon [30], and scallops [31]. 2-Ethylpyridine was found to be closely associated with claw meat, which might be affected 
by its relatively higher intensity score in the GC-O study. Naphthalene and 2-methylnaphthalene, which may be considered as products generated from environmental contaminants [30], have been previously reported in crabs [12] and fresh salmon [30]. As numerous of ODCs had been found in four edible parts of $E$. sinensis (most of them contributed to pleasant aroma), a limited negative impact could be made by naphthalene and 2-methylnaphthalene to the integral aroma of $E$. sinensis.

The aroma in leg meat was moderate, which may be caused by the lower quantities of IOCs. (Z)-4-Heptenal, 2-pentylfuran, 2-nonanone, and 2-decanone had a comparatively high odor intensity in leg meat than the other three parts. Among these ODCs, (Z)-4-heptenal and 2-pentylfuran showed a close relationship with leg meat (Fig. 1). (Z)-4-Heptenal is usually considered as the degradation products of linoleate and linolenate hydroperoxides [7, 26]. The percentage of n-3 PUFAs in leg meat was relatively higher $(37.33 \%)$ than the other three parts [24], which may explain the high intensity of (Z)-4-heptenal in leg meat. 2-Pentylfuran has not been detected as ODC in E. sinensis before, but it presented a "sweet,green" aroma in crabs farmed in the Songjiang district. The estimated concentration in leg meat was much higher than that of in the other three parts. These compounds could be produced by thermal degradation of lipid or thiamine [32].

In summary, the aroma profiles of four edible parts of Chinese mitten crab were composed of 39 ODCs found by GC-MS/O. The ODCs of Chinese mitten crab were dominated by the presence of numerous aldehydes and N-containing compounds. Among them, IOCs would make a great contribution to aroma profiles of each part. 2-Ethylpyridine, trimethylamine, benzaldehyde, 2,5-dimethylpyrazine, 2,3-dimethylpyrazine, and 1-penten-3-ol were found to be IOCs in no less than two edible parts of $E$. sinensis. The differences of aroma properties from four edible parts were detected by E-Nose and sensory evaluation. The gonad part had strong aroma in ammonia-like, fishy, grassy, and fatty attributes in sensory evaluation, which make it differentiate from the other three parts. Hexanal and 2-acetylthiazole might be the important contributors to the aroma profile of the gonad. Abdomen meat and claw meat were related to a strong meat aroma. 3-Methyl-2-thiophenecarboxaldehyde could make a great contribution to the aroma profile of abdomen meat, while 2-ethylpyridine could be associated closely with the aroma profile of claw meat. The aroma of leg meat was moderate, and (Z)4-heptenal might be the important contributor to the leg meat.

\footnotetext{
Acknowledgments This work was supported by Grants from the Industrial System Project of the Chinese Mitten Crab of Shanghai Municipal Agriculture Commission (Grant No. D-8003-10-0208), the Leading Academic Discipline Project of the Shanghai Municipal Education Commission (Project No. J50704), and the National Natural Science Foundation of China (Grant No. 31471608).
}

\section{Compliance with ethics standards}

Conflict of interest Siru Ji, Saiqi Gu, Xichang Wang, and $\mathrm{Na} \mathrm{Wu}$ declare that they have no conflict of interest.

Human or animal subjects This article does not contain any studies with human or animal subjects.

Open Access This article is distributed under the terms of the Creative Commons Attribution 4.0 International License (http://creativecommons.org/licenses/by/4.0/), which permits unrestricted use, distribution, and reproduction in any medium, provided you give appropriate credit to the original author(s) and the source, provide a link to the Creative Commons license, and indicate if changes were made.

\section{References}

1. Rudnick DA, Hieb K, Grimmer KF (2003) Patterns and processes of biological invasion: the Chinese mitten crab in San Francisco Bay. Basic Appl Ecol 4:249-262

2. China Fishery Bureau (2013) China fisheries yearbook. Chinese Agriculture, Beijing (in Chinese)

3. Wen XB, Chen LQ, Ai CX, Zhou ZL, Jiang HB (2001) Variation in lipid composition of Chinese mitten-handed crab, Eriocheir sinensis during ovarian maturation. Comp Biochem Phys B 130:95-104

4. Chen DW, Zhang M (2007) Non-volatile taste active compounds in the meat of Chinese mitten crab (Eriocheir sinensis). Food Chem 104:1200-1205

5. Chen DW, Zhang M, Shrestha S (2007) Compositional characteristics and nutritional quality of Chinese mitten crab (Eriocheir sinensis). Food Chem 103:1343-1349

6. Kong L, Cai CF, Ye YT, Chen DX, Wu P, Li EC (2012) Comparison of non-volatile compounds and sensory characteristics of Chinese mitten crabs (Eriocheir sinensis) reared in lakes and ponds: potential environmental factors. Aquaculture 364:96-102

7. Gu SQ, Wang XC, Tao NP, Wu N (2013) Characterization of volatile compounds in different edible parts of steamed Chinese mitten crab (Eriocheir sinensis). Food Res Int 54:81-92

8. Chung H, Cadwallader K (1994) Aroma extract dilution analysis of blue crab claw meat volatiles. J Agric Food Chem 42:2867-2870

9. Yu HZ, Chen SS (2010) Identification of characteristic aromaactive compounds in steamed mangrove crab (Scylla serrata). Food Res Int 43:2081-2086

10. Niu Y, Zhang X, Xiao Z, Song S, Eric K, Jia C, Yu H, Zhu J (2011) Characterization of odor-active compounds of various cherry wines by gas chromatography-mass spectrometry, gas chromatography-olfactometry and their correlation with sensory attributes. J Chromatogr B 879:2287-2293

11. Chen DW, Zhang M (2010) Determination of odor-active compounds in the cooked meat of Chinese mitten crab (Eriocheir Sinensis) by solid phase microextraction, gas chromatographyolfactometry and gas chromatography-mass spectrometry. J Food Drug Anal 18:290-296

12. Wu N, Gu SQ, Tao NP, Wang XC, Ji SR (2014) Characterization of important odorants in steamed male Chinese Mitten Crab (Eriocheir sinensis) using Gas Chromatography-Mass Spectrometry-Olfactometry. J Food Sci 79:1250-1259

13. Pollien P, Ott A, Montigon F, Baumgartner M, Muñoz-Box R, Chaintreau A (1997) Hyphenated headspace-gas chromatographysniffing technique: screening of impact odorants and quantitative aromagram comparisons. J Agric Food Chem 45:2630-2637 
14. Lu FSH, Bruheim I, Haugsgjerd BO, Jacobsen C (2014) Effect of temperature towards lipid oxidation and non-enzymatic browning reactions in krill oil upon storage. Food Chem 157:398-407

15. Lu F, Zhang J-Y, Liu S-L, Wang Y, Ding Y-T (2011) Chemical, microbiological and sensory changes of dried Acetes chinensis during accelerated storage. Food Chem 127:159-168

16. Elmore JS, Mottram DS, Enser M, Wood JD (2000) The effects of diet and breed on the volatile compounds of cooked lamb. Meat Sci 55:149-159

17. Guo YR, Gu SQ, Wang XC, Zhao LM, Zheng JY (2014) Comparison of fatty acid and amino acid profiles of steamed Chinese mitten crab. Fish Sci 80:621-633

18. Lee G-H, Suriyaphan O, Cadwallader KR (2001) Aroma components of cooked tail meat of American lobster (Homarus americanus). J Agric Food Chem 49:4324-4332

19. Olafsdottir G, Jonsdottir R, Lauzon HL, Luten J, Kristbergsson K (2005) Characterization of volatile compounds in chilled cod (Gadus morhua) fillets by gas chromatography and detection of quality indicators by an electronic nose. J Agric Food Chem 53:10140-10147

20. Wu W, Tao NP, Gu SQ (2014) Characterization of the key odoractive compounds in steamed meat of Coilia ectenes from Yangtze River by GC-MS-O. Eur Food Res Technol 238:237-245

21. Dominguez R, Gómez M, Fonseca S, Lorenzo JM (2014) Effect of different cooking methods on lipid oxidation and formation of volatile compounds in foal meat. Meat Sci 97:223-230

22. Chung HY, Yeung CW, Kim JS, Chen F (2007) Static headspace analysis-olfactometry (SHA-O) of odor impact components in salted-dried white herring (Ilisha elongata). Food Chem 104:842-851

23. Wong J, Bernhard R (1988) Effect of nitrogen-source on pyrazine formation. J Agric Food Chem 36:123-129
24. Chen DW, Zhang M, Shrestha S (2007) Compositional characteristics and nutritional quality of Chinese mitten crab (Eriocheir sinensis). Food Chem 103:1343-1349

25. Kong L, Cai CF, Ye YT, Chen DX, Wu P, Li EC, Chen LQ, Lin S (2012) Comparison of non-volatile compounds and sensory characteristics of Chinese mitten crabs (Eriocheir sinensis) reared in lakes and ponds: potential environmental factors. Aquaculture 364-365:96-102

26. Grosch W (1982) In: Morton ID, Macleod AJ (eds) Lipid degradation products and flavor. Elsevier, Amsterdam, p 325

27. Alasalvar C, Taylor KA, Shahidi F (2005) Comparison of volatiles of cultured and wild sea bream (Sparus aurata) during storage in ice by dynamic headspace analysis/gas chromatographymass spectrometry. J Agric Food Chem 53:2616-2622

28. Osorio MT, Zumalacárregui JM, Cabeza EA, Figueira A, Mateo J (2008) Effect of rearing system on some meat quality traits and volatile compounds of suckling lamb meat. Small Ruminant Res 78:1-12

29. Le Guen S, Prost C, Demaimay M (2000) Characterization of odorant compounds of mussels (Mytilus edulis) according to their origin using gas chromatography-olfactometry and gas chromatography-mass spectrometry. J Chromatogr B 896:361-371

30. Varlet V, Knockaert C, Prost C, Serot T (2006) Comparison of odor-active volatile compounds of fresh and smoked salmon. J Agric Food Chem 54:3391-3401

31. Chung HY, Yung IKS, Ma WCJ, Kim J (2002) Analysis of volatile components in frozen and dried scallops (Patinopecten yessoensis) by gas chromatography/mass spectrometry. Food Res Int 35:43-53

32. Vernin G, Parkanyi C (1982) In: Vernin G (ed) Mechanisms of formation of heterocyclic compounds in Maillard and pyrolysis reactions. Ellis Horwood, Chichester, pp 151-207 